\title{
On Controller Initialization in Multivariable Switching Systems *
}

\author{
Farshad R. Pour Safaei ${ }^{\text {a,* }}$, João P. Hespanha ${ }^{a}$, Gregory Stewart ${ }^{\text {b }}$

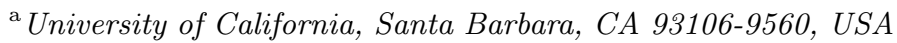 \\ ${ }^{\mathrm{b}}$ Honeywell, 500 Brooksbank Avenue, North Vancouver, BC Canada V7J $3 S_{4}$
}

\begin{abstract}
We consider a class of switched systems which consists of a linear MIMO and possibly unstable process in feedback interconnection with a multicontroller whose dynamics switch. It is shown how one can achieve significantly better transient performance by selecting the initial condition for every controller when it is inserted into the feedback loop. This initialization is obtained by performing the minimization of a quadratic cost function of the tracking error, controlled output, and control signal. We guarantee input-to-state stability of the closed-loop system when the average number of switches per unit of time is smaller than a specific value. If this is not the case then stability can still be achieved by adding a mild constraint to the optimization. We illustrate the use of our results in the control of a flexible beam actuated in torque. This system is unstable with two poles at the origin and contains several lightly damped modes, which can be easily excited by controller switching.
\end{abstract}

Key words: Switching controllers, Linear systems, Multi-input/multi-output systems, Quadratic performance indices.

\section{Introduction}

The classical approach to satisfy multiple conflicting requirements in a linear control system has some inherent limitations that have been well recognized in the literature, Boyd \& Barrat (1991). A solution to such problems is to design several controllers with distinct transfer functions $\left\{K_{q}(s): q \in \mathcal{P}\right\}$, and switch between them. Examples of such systems are the fault tolerant control, Efimov et al. (2010a), and the gain scheduling problem, Stewart (2012). To achieve the best overall performance, it is the job of the designer to choose which controller to use at each time instant, based on the current operating conditions (e.g. Al-Shyoukh \& Shamma (2009)).

In designing a multicontroller in a linear switching system, there are several degrees of freedom available to de-

\footnotetext{
* This research was partially supported by the NSF Grant CNS-0720842. The material in this paper was partially presented at 2010 American Control Conference, Baltimore, MD.

* Corresponding author. Tel.: +1 805893 7785; fax: +1 805 8933262.

Email addresses: farshad@ece.ucsb.edu (Farshad R. Pour Safaei ), hespanha@ece.ucsb.edu ( João P. Hespanha), greg. Stewart@Honeywell.com (Gregory Stewart).
}

signers. Two of them, selecting different controller transfer functions and deciding which controller to use at each instant of time, are widely used. In addition, one can take advantage of another degree of freedom which is rarely used by designers: selecting suitable initial conditions for the controllers when they are inserted into the feedback loop. We mainly focus on exploring this degree of freedom to increase the performance of the overall system. Moreover, since stability of switching systems depends on controller realization, by choosing appropriate statespace realizations for controller transfer functions, we guarantee closed-loop stability under very mild assumptions on the switching mechanism. We assume that the set of controller transfer functions is given and that we have no control over the mechanism by which it is decided when the different controllers are switched in and out of the feedback loop.

Switching between controllers has the potential to cause adverse transient responses and, consequently, the mitigation of such transients has been addressed in the literature. Turner \& Walker (2000) proposed to address this by minimizing the difference between the control signal that is being fed to the process and the control signals produced by out-of-loop controllers. This minimization was formulated as a linear quadratic (LQ) optimization and resulted in a static feedback law for the out-of-loop con- 
trollers. Based on this work, Zheng \& Benstman (2009) and Zheng et al. (2007) have analyzed the steady-state gain of the infinite horizon LQ bumpless transfer topologies and steady state bumpless control transfer under uncertainties. This type of approach is motivated by the intuitive appeal of "bumpless transfer:" by switching into the feedback loop a controller whose output is close to the current control signal, the transient should be small. Here, we consider a more direct approach in that we will directly address the problem of improving transient performance of the system, without making assumption on whether or not bumpless transfer will lead to a better transient. We will show that transients due to switching can be mostly suppressed by carefully initializing the state of a new controller to be inserted into the feedback loop, so as to minimize a quadratic cost that combines the control signal, error signal and controlled output.

Turner \& Walker (2000) assumed that both on- and off-line controllers are stabilizing for the plant in question however nothing can be concluded about stability of the overall system under arbitrary switching, Liberzon (2003). Hespanha \& Morse (2002) considered the problem of selecting controller realizations and choosing initial conditions to stabilize a switched system, but no improvement in the transient response of the switching controller was considered. The optimal control problem for switched systems was considered in Xu \& Antsaklis (2004) where they assume that a prespecified sequence of active subsystems is given and optimal switching times are driven. Also Efimov et al. (2010b) derive a switching law to improve the performance under stabilizing controller. Moreover, Stewart \& Dumont (2006) presents a technique to improve the performance of each subcontroller in discrete time systems which is further pursued in Hespanha et al. (2007) for controller initialization.

The specific problem formulated here was first introduced in Hespanha et al. (2007), which provided a method to select controller realizations and initial conditions for the case of an asymptotically stable SISO plant to be controlled. The stability results of Hespanha et al. (2007) were restricted to the case of piecewise constant reference signals. The main contribution of this paper is to extend these results to MIMO, possibly unstable processes and show that it is possible to obtain input-to-state stability (ISS) of the closed-loop for arbitrary references. In particular, we show that ISS can be obtained through two alternative mechanisms: when the average number of switches per unit of time is smaller than a specific value, the closed-loop system remains ISS. If this is not the case then the ISS property can still be achieved by adding a mild constraint to the optimization and to select the initial controller state.

The remainder of this paper is organized as follows. In section 2 , the mathematical problem statement is intro- duced. Section 3 shows how to choose the controller reset map to minimize a quadratic criteria. Section 4 proves the stability of the closed loop under a mild assumption on the switching signal. Simulation results that compare the performance of our switching controller with those in Hespanha \& Morse (2002) for a MIMO unstable process are provided in sections 5 . Finally, section 6 provides conclusions and directions for future work.

\section{Problem statement}

\subsection{Controller architecture}

The switched systems considered here arises from the feedback interconnection of a Linear Time-Invariant (LTI) plant $\Sigma$ to be controlled with a multicontroller $\mathbf{C}(\sigma)$ whose inputs are the usual tracking error $e_{T}(t)$ as well as a piecewise constant switching signal $\sigma:[0,+\infty) \rightarrow \mathcal{P}$ that determines which controller transfer function to use at each time instant.

More specifically, when $\sigma(t)$ is set constant equal to some $q \in \mathcal{P}$, the multicontroller should behave as an LTI system with transfer function $K_{q}(s)$. In particular, given $n$-dimensional state space realizations $E_{q}, F_{q}, V_{q}, W_{q}$ for each $K_{q}(s), q \in \mathcal{P}$, the state $x_{\text {mult }}(t)$ of the multicontroller $\mathbf{C}(\sigma)$ evolves according to

$$
\left\{\begin{array}{l}
\dot{x}_{\text {mult }}(t)=E_{\sigma(t)} x_{\text {mult }}(t)+F_{\sigma(t)} e_{T}(t) \\
u(t)=V_{\sigma(t)} x_{\text {mult }}(t)+W_{\sigma(t)} e_{T}(t)
\end{array}\right.
$$

on any time interval on which the switching signal $\sigma(t)$ remains constant, and according to

$$
x_{\text {mult }}(t)=\psi\left(x_{\text {mult }}\left(t^{-}\right), \sigma\left(t^{-}\right), \sigma(t), r(t)\right),
$$

at every time $t$, called a switching time, at which $\sigma(t)$ is discontinuous. The function $\psi($.$) is called the reset map$ and it determines the initial state of the controllers at the switching times. We let such initial state depend on the state $x_{\text {mult }}\left(t^{-}\right)$of the controller just before the switching time, the index $p$ of the previous controller $\left(\sigma\left(t^{-}\right)=p\right)$, the index $q$ of the new controller $(\sigma(t)=q)$, and also on the current reference value $r(t)$. Our goal is to select the reset map so as to achieve a smooth transient at the controller switching times. In particular, we select this function so as to minimize a quadratic cost function that penalizes the tracking error, the controlled output, and the control signal, all integrated over an interval that starts at the switching time.

The construction of the multicontroller follows Hespanha (2009) and is inspired by the Q-parameterization of all the stabilizing controllers, Youla et al. (1976). In this parameterization, one can define the set of all possible stabilizing controllers as a function of the so-called 
parameter $Q$ which is a stable transfer matrix. This is a useful tool in problems where one wishes to optimize a performance objective with a stability constraint.

Consider a multivariable LTI process $\Sigma$ with transfer function $\mathrm{G}_{\mathrm{pl}}(s)$ from the input $u(t)$ to the output $y(t)$, and assume given a finite family of stabilizing controller transfer functions $\left\{K_{q}(s): q \in \mathcal{P}\right\}$ from the tracking error $e_{T}(t)$ to the control input $u(t)$, where $r(t)$ denotes a reference signal.

Let $(A, B, C)$ denote a stabilizable and detectable $n_{\mathrm{pl}^{-}}$ dimensional realization for the process transfer function $\mathrm{G}_{\mathrm{pl}}(s)$ :

$$
\dot{x}_{\mathrm{pl}}=A x_{\mathrm{pl}}+B u, \quad y=C x_{\mathrm{pl}}, \quad z=G x_{\mathrm{pl}}+H u
$$

where $x_{\mathrm{pl}} \in \mathbb{R}^{n_{\mathrm{pl}}}$ denotes the process state, $u \in \mathbb{R}^{k}$ the control signal, $y \in \mathbb{R}^{m}$ the measured output and $z \in \mathbb{R}^{l}$ the controlled output. The measured output $y(t)$ corresponds to the signal that is available for control whereas $z(t)$ corresponds to the signal that one would like to make as small as possible in the shortest possible time. It should be noted that matrix the matrix $H$ represents a direct feedthrough term between $u$ and controlled output $z$. Such matrix is needed if we want to penalize the control signal. One could also consider a similar feedthrough term between $u$ and the measured output $y$. For simplicity, we are ignoring such term. If such term exists, we can get similar results by applying the parameterization techniques introduced in (Dullerud \& Paganini, 1999, Chapter 5).

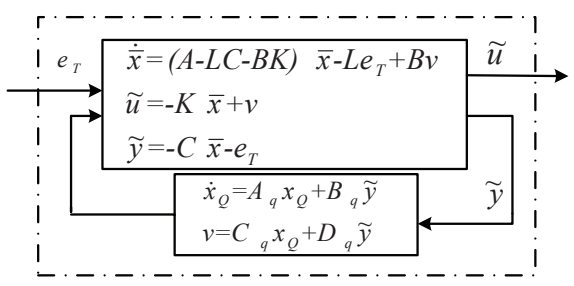

Fig. 1. Controller $K_{q}(s)$

As stated in the following theorem, one can select an LQG/LQR Q-augmented realization for each stabilizing controller of the form shown in Figure 1.

Theorem (Q-parametrization) 2.1 Assume that $A-L C$ and $A-B K$ are Hurwitz matrices. For every controller transfer matrix $K_{q}(s)$ that asymptotically stabilizes (3), there exists a BIBO (Bounded-InputBounded-Output) stable transfer matrix $Q_{q}(s)$ such that for every stabilizable and detectable realization $\left(A_{q}, B_{q}, C_{q}, D_{q}\right)$ of $Q_{q}(s)$, the controller transfer matrix $K_{q}(s)$ admits a stabilizable and detectable realization

$$
\left(E_{q}, F_{q}, V_{q}, W_{q}\right) \text { with }
$$

$$
\begin{aligned}
E_{q} & :=\left[\begin{array}{rr}
A-L C-B K-B D_{q} C & B C_{q} \\
-B_{q} C & A_{q}
\end{array}\right], \\
F_{q} & :=\left[\begin{array}{c}
-L-B D_{q} \\
-B_{q}
\end{array}\right], \\
V_{q} & :=\left[\begin{array}{ll}
-K-D_{q} C & C_{q}
\end{array}\right], \quad W_{q}:=-D_{q}
\end{aligned}
$$

where $A_{q}$ matrices are selected so that $A_{q}+A_{q}^{\prime}<0$.

The controller realization, introduced in Theorem 2.1, corresponds to the diagram in Figure 1.

Proof of Theorem 2.1 The existence of $Q$ system and realizations (4)-(5) are proved in Hespanha (2009). We further show that one can always find $A_{q}$ matrices that satisfy

$$
A_{q}+A_{q}^{\prime}<0, \quad \forall q \in \mathcal{P} .
$$

Consider a finite family of Hurwitz matrices $\mathcal{S}=\left\{\tilde{\mathcal{A}}_{q}\right.$ : $q \in \mathcal{P}\}$. By using a similarity transformation, we show how to compute $A_{q}$ matrices for every $\tilde{\mathcal{A}}_{q}$ such that it satisfies (6). Since $\tilde{\mathcal{A}}_{q}$ matrices are asymptotically stable, the family of Lyapunov equations

$$
\mathcal{Q}_{q} \tilde{\mathcal{A}}_{q}+\tilde{\mathcal{A}}_{q}^{\prime} \mathcal{Q}_{q}=-I \quad q \in \mathcal{P}
$$

always have positive definite symmetric solutions $\mathcal{Q}_{q}$ which can be written as $\mathcal{Q}_{q}=Y_{q}^{\prime} Y_{q}$. Let $A_{q}=Y_{q} \tilde{\mathcal{A}}_{q} Y_{q}^{-1}$. From (7), we can conclude that

$$
Y_{q}^{\prime}\left(A_{q}+A_{q}^{\prime}\right) Y_{q}=-I
$$

which yields to $A_{q}+A_{q}^{\prime}=-\left(Y_{q}^{\prime}\right)^{-1}\left(Y_{q}\right)^{-1}<0$.

We now propose to use a multicontroller $C(\sigma)$ with state $x_{\text {mult }}=\left[\bar{x}^{\prime}, x_{Q}^{\prime}\right]^{\prime}$ that evolves according to (1), with realization (4)-(5), on any time interval on which the switching signal $\sigma(t)$ remains constant and is reset according to the equation (2) at every switching time $t$. In (2), only the $x_{Q}$ component of the state of the multi-controller is reset according to a map $\psi_{Q}($.$) to be defined in section 3$.

\subsection{Closed-loop configuration}

The optimal set point problem can be reduced to that of optimal regulation by considering an auxiliary system with state $\tilde{x}:=x-x_{e q}$, [cf. Hespanha (2009)]. This motivates us to consider the closed-loop configuration as shown in Figure 2 with appropriately defined matrices $N$ and $F$. If one wants the controlled output $z$ to converge to a given nonzero constant set-point value $r \in \mathbb{R}^{l}$, there 


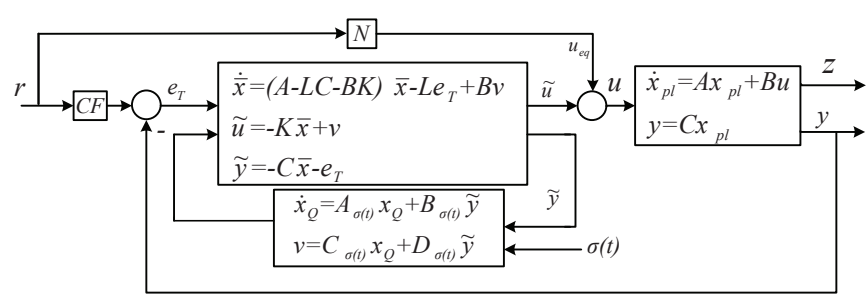

Fig. 2. Closed-loop Architecture

should exist an equilibrium point $\left(x_{e q}, u_{e q}\right)$ that satisfies the linear equation

$$
\left[\begin{array}{cc}
-A & B \\
-G & H
\end{array}\right]\left[\begin{array}{c}
-x_{e q} \\
u_{e q}
\end{array}\right]=\left[\begin{array}{c}
0 \\
r
\end{array}\right] \quad x_{e q} \in \mathbb{R}^{n_{\mathrm{pl}}}, u_{e q} \in \mathbb{R}^{k}
$$

Depending on the number of inputs $k$ and the number of controlled outputs $l$, three different cases should be considered: ( $i) k<l$ (under-actuated system) for which (8) generally does not have a solution (ii) $k>l$ (overactuated system) for which (8) generally has more than one solution (iii) $k=l$ for which (8) always has a solution as long as the coefficient matrix in (8) is nonsingular. If there exists a solution to (8), it can be written in the form $x_{e q}=F r, u_{e q}=N r$ for appropriately defined matrices $F$ and $N$, which corresponds to the control architecture in Figure 2. The feed-forward term $N r$ is absent when the state matrix $A$ has an eigen value at the origin and this mode is observable through $z$. Note that in this case the process has an integrator. Also, when $z=y$, we have $G=C, H=0$, and in this case $C x_{e q}=r$. This corresponds to $C F=1$.

We are now ready to find the closed-loop realization of the system shown in Figure 2. Connecting the plant $\Sigma$ with realization $(A, B, C)$ to the multicontroller $\mathbf{C}(\sigma)$, through the negative feedback interconnection, results in a switched system with a state $x(t)=\left[x_{\mathrm{pl}}(t)^{\prime}, \bar{x}(t)^{\prime}, x_{Q}(t)^{\prime}\right]^{\prime}$ that evolves according to

$$
\begin{aligned}
\dot{x}(t) & =\hat{A}_{\sigma(t)} x(t)+\hat{B}_{\sigma(t)} r(t), \\
y(t) & =\hat{C}_{\sigma(t)} x(t), \\
z(t) & =\hat{G}_{\sigma(t)} x(t)+\hat{H}_{\sigma(t)} r(t)
\end{aligned}
$$

on any time interval on which $\sigma(t)$ remains constant and

$$
x(t)=\left[\begin{array}{c}
x_{\mathrm{pl}}(t) \\
\bar{x}(t) \\
x_{Q}(t)
\end{array}\right]=\left[\begin{array}{c}
x_{\mathrm{pl}}\left(t^{-}\right) \\
\bar{x}\left(t^{-}\right) \\
\psi_{Q}\left(x\left(t^{-}\right), \sigma\left(t^{-}\right), \sigma(t), r(t)\right)
\end{array}\right]
$$

at every switching time $t$. The matrices in (9) are defined by

$$
\begin{aligned}
& \hat{A}_{q}:=\left[\begin{array}{ccc}
A+B D_{q} C & -B\left(K+D_{q} C\right) & B C_{q} \\
\left(L+B D_{q}\right) C & A-L C-B K-B D_{q} C & B C_{q} \\
B_{q} C & -B_{q} C & A_{q}
\end{array}\right], \\
& \hat{B}_{q}:=\left[\begin{array}{c}
B N-B D_{q} C F \\
-\left(L+B D_{q}\right) C F \\
-B_{q} C F
\end{array}\right] \\
& \hat{C}_{q}:=\left[\begin{array}{lll}
C & 0 & 0
\end{array}\right] \text {, } \\
& \hat{G}_{q}:=\left[G+H D_{q} C H\left(-K-D_{q} C\right) C_{q}\right] \text {, } \\
& \hat{H}_{q}:=H\left(N-D_{q} C F\right), \quad \forall q \in \mathcal{P} \text {. }
\end{aligned}
$$

Since each controller transfer function $K_{q}(s)$ asymptotically stabilizes the process, all $\hat{A}_{q}$ matrices are Hurwitz.

In the remainder of this paper, we focus on the goal of selecting an appropriate reset map $\psi_{Q}(\cdot)$ in (10) that achieves an optimal transient performance at switching times, while maintaining the stability of the closed-loop system. The idea of optimizing transient performance is the subject of the next section.

\section{Optimization of transient performance}

\subsection{Quadratic cost function}

As discussed in previous section, only $x_{Q}(t)$ component of (10) jumps at switching times, and the other two components evolve continuously. Suppose that there is a switching at $t=t_{0}$, which results in a jump in the switching signal from $\sigma\left(t_{0}^{-}\right)=p$ to $\sigma\left(t_{0}\right)=q$. In what follows, we try to select an appropriate post-switching state defined by the reset map

$$
x_{Q}\left(t_{0}\right)=\psi_{Q}\left(x\left(t_{0}^{-}\right), p, q, r\left(t_{0}\right)\right)
$$

such that it optimizes the resulting transient performance, as measured by a quadratic cost of the following form

$$
\begin{aligned}
J= & \int_{t_{0}}^{t_{1}}\left(e_{T}(t)^{\prime} R e_{T}(t)+z(t)^{\prime} W z(t)+u(t)^{\prime} K u(t)\right) d t \\
& +\left(x\left(t_{1}\right)-x_{\infty}\right)^{\prime} T\left(x\left(t_{1}\right)-x_{\infty}\right)
\end{aligned}
$$

where $e_{T}, z$ and $u$ are shown in Figure 2. In the above equation, $t_{1}$ is a time larger than $t_{0}$ and possibly equal to $+\infty ; R, W, K, T$ are appropriately selected positive semidefinite symmetric matrices, and $x_{\infty}:=-\hat{A}_{q}^{-1} \hat{B}_{q} r\left(t_{0}\right)$. Note that the vector $x_{\infty}$ that appears in the terminal term in (12) is the steady-state value to which $x(t)$ would converge as $t \rightarrow \infty$ if both $\sigma(t)$ and $r(t)$ were to remain constant. Moreover, It is worth noting that all the results 
in this section hold for an infinite horizon, as we make $t_{1} \rightarrow \infty$ in (12), in which case the terminal cost term in (12) disappears.

One can define a transient performance by appropriately defining $R, W, K, T$ matrices in (12) which correspond to penalizing the tracking error, controlled output, control effort, and final state magnitude, respectively. In this optimization, we assume that the switching signal $\sigma(t)$ and the reference $r(t)$ remain constant and equal to $q$ and $r\left(t_{0}\right)$ over the optimization horizon $\left[t_{0}, t_{1}\right]$. If $\sigma(t)$ turns out to switch again before $t_{1}$, the value to which $x_{Q}$ was reset at time $t_{0}$ will generally not be optimal, but in section 4 we will make sure that stability is preserved even if unexpected switches occur.

\subsection{Optimal reset map}

To find the value of $x_{Q}\left(t_{0}\right)$ in (11) that minimizes (12) we need to introduce the following notation: Let $Q_{q}$ denote the symmetric solution to the following Lyapunov equation

$$
Q_{q} \hat{A}_{q}+\hat{A}_{q}^{\prime} Q_{q}=-P_{q},
$$

where $P_{q}:=\hat{C}_{q}^{\prime} R \hat{C}_{q}+\hat{G}_{q}^{\prime} W \hat{G}_{q}+\tilde{C}_{q}^{\prime} K \tilde{C}_{q} \geq 0$ and $\tilde{C}_{q}:=$ $\left[D_{q} C-\left(K+D_{q} C\right) C_{q}\right]$. Such $Q_{q}$ always exists and is positive semi-definite because $\hat{A}_{q}$ is a Hurwitz matrix. We also define the positive semi-definite matrix $M_{q}:=$ $Q_{q}+e^{\hat{A}_{q}^{\prime} \Delta}\left(T-Q_{q}\right) e^{\hat{A}_{q} \Delta}$, the length of optimization interval $\Delta:=t_{1}-t_{0}$, and the vector

$$
\begin{aligned}
g_{q}^{\prime} & :=2 r\left(t_{0}\right)^{\prime}\left(\left(-F^{\prime} C^{\prime} R \hat{C}_{q}+\hat{H}_{q}^{\prime} W \hat{G}_{q}+\right.\right. \\
& \left.\left(N-D_{q} C F\right)^{\prime} K \tilde{C}_{q}+\hat{B}_{q}^{\prime} Q_{q}\right) \hat{A}_{q}^{-1}\left(I-e^{\hat{A}_{q} \Delta}\right) \\
& \left.+\hat{B}_{q}^{\prime}\left(e^{\hat{A}_{q}^{\prime} \Delta}\left(\hat{A}_{q}^{\prime}\right)^{-1}\left(Q_{q}-T\right)-\left(\hat{A}_{q}^{\prime}\right)^{-1} Q_{q}\right) e^{\hat{A}_{q} \Delta}\right) .
\end{aligned}
$$

We further need to block-partition the symmetric matrix $M_{q}$ and the vector $g_{q}$ according to the partition in (10) of the state vector:

$$
M_{q}=\left[\begin{array}{ccc}
M_{11}^{q} & M_{12}^{q} & M_{13}^{q} \\
M_{21}^{q} & M_{22}^{q} & M_{23}^{q} \\
M_{31}^{q} & M_{32}^{q} & M_{33}^{q}
\end{array}\right], \quad g_{q}=\left[\begin{array}{c}
g_{1}^{q} \\
g_{2}^{q} \\
g_{3}^{q}
\end{array}\right]
$$

and perform a singular value decomposition of

$$
M_{33}^{q}=\left[\begin{array}{ll}
U_{1}^{q} & U_{2}^{q}
\end{array}\right]\left[\begin{array}{cc}
\Lambda_{q} & 0 \\
0 & 0
\end{array}\right]\left[\begin{array}{l}
\left(V_{1}^{q}\right)^{\prime} \\
\left(V_{2}^{q}\right)^{\prime}
\end{array}\right]
$$

(with $\Lambda_{q}$ nonsingular). We are now ready to provide the solution to the above quadratic optimization problem.

Theorem 3.1 Assume that there is a single switching at $t=t_{0}$, where $\sigma\left(t_{0}^{-}\right)=p, \sigma\left(t_{0}\right)=q$ and $r(t)=r\left(t_{0}\right)$ $\forall t \in\left[t_{0}, t_{1}\right]$. The reset map (with the smallest Euclidean norm)

$$
\begin{aligned}
x_{Q}^{*}\left(t_{0}\right) & =V_{1}^{q} \Lambda_{q}^{-1}\left(U_{1}^{q}\right)^{\prime} . \\
& \left(\frac{1}{2} g_{3}^{q}-\left(M_{13}^{q}\right)^{\prime} x_{\mathrm{pl}}\left(t_{0}^{-}\right)-M_{32}^{q} \bar{x}\left(t_{0}^{-}\right)\right) .
\end{aligned}
$$

provides a global minimum to (12).

Proof of Theorem 3.1 We first compute the quadratic cost function $J$ along a solution to the closed loop dynamics in the optimization interval:

$$
\begin{aligned}
& x(t)=e^{\hat{A}_{q}\left(t-t_{0}\right)} x\left(t_{0}\right)+\int_{t_{0}}^{t} e^{\hat{A}_{q}(t-\tau)} \hat{B}_{q} \cdot r\left(t_{0}\right) d \tau, \\
& u(t)=\tilde{C}_{q} x(t)+\left(N-D_{q} C F\right) r(t), \quad \forall t \in\left[t_{0}, t_{1}\right] .
\end{aligned}
$$

Straightforward algebra shows that the terminal term in (12) is given by

$$
\begin{aligned}
& \left(x\left(t_{1}\right)-x_{\infty}\right)^{\prime} T\left(x\left(t_{1}\right)-x_{\infty}\right)=\left[e^{\hat{A}_{q}\left(t_{1}-t_{0}\right)} x\left(t_{0}\right)+\right. \\
& \quad \int_{t_{0}}^{t_{1}} e^{\hat{A}_{q}\left(t_{1}-\tau\right)} \hat{B}_{q} \cdot r\left(t_{0}\right) d \tau+ \\
& \left.\quad+\hat{A}_{q}^{-1} \hat{B}_{q} r\left(t_{0}\right)\right]^{\prime} T\left[e^{\hat{A}_{q}\left(t_{1}-t_{0}\right)} x\left(t_{0}\right)+\right. \\
& \left.\quad+\int_{t_{0}}^{t_{1}} e^{\hat{A}_{q}\left(t_{1}-\tau\right)} \hat{B}_{q} \cdot r\left(t_{0}\right) d \tau+\hat{A}_{q}^{-1} \hat{B}_{q} r\left(t_{0}\right)\right] \\
& \quad=x\left(t_{0}\right)^{\prime} e^{\hat{A}_{q}^{\prime} \Delta} T e^{\hat{A}_{q} \Delta} x\left(t_{0}\right) \\
& \quad+2 r\left(t_{0}\right)^{\prime} \hat{B}_{q}^{\prime} e^{\hat{A}_{q}^{\prime} \Delta}\left(\hat{A}_{q}^{-1}\right)^{\prime} T e^{\hat{A}_{q} \Delta} x\left(t_{0}\right)+r\left(t_{0}\right)^{\prime} . \\
& \\
& \quad \hat{B}_{q}^{\prime}\left(e^{\hat{A}_{q}^{\prime} \Delta}-I\right)\left(\hat{A}_{q}^{-1}\right)^{\prime} T \hat{A}_{q}^{-1}\left(e^{\hat{A}_{q} \Delta}-I\right) \hat{B}_{q} r\left(t_{0}\right) \\
& \quad+r\left(t_{0}\right)^{\prime} \hat{B}_{q}^{\prime}\left(\hat{A}_{q}^{-1}\right)^{\prime} T \hat{A}_{q}^{-1}\left(e^{\hat{A}_{q} \Delta}-\frac{1}{2} I\right) \hat{B}_{q} r\left(t_{0}\right) .
\end{aligned}
$$

Since

$$
\begin{gathered}
e_{T}(t)^{\prime} R e_{T}(t)=r\left(t_{0}\right)^{\prime} F^{\prime} C^{\prime} R C F r\left(t_{0}\right) \\
-2 r\left(t_{0}\right)^{\prime} F^{\prime} C^{\prime} R \hat{C}_{q} x(t)+x(t)^{\prime} \hat{C}_{q}^{\prime} R \hat{C}_{q} x(t), \\
u(t)^{\prime} K u(t)=x(t)^{\prime} \tilde{C}_{q}^{\prime} K \tilde{C}_{q} x(t) \\
+2 r\left(t_{0}\right)^{\prime}\left(N^{\prime}-F^{\prime} C^{\prime} D_{q}^{\prime}\right) K \tilde{C}_{q} x(t) \\
+r\left(t_{0}\right)\left(N^{\prime}-F^{\prime} C^{\prime} D_{q}^{\prime}\right) K\left(N-D_{q} C F\right) r\left(t_{0}\right), \\
z(t)^{\prime} W z(t)=\left(\hat{G}_{q} x(t)+\hat{H}_{q} r\left(t_{0}\right)\right)^{\prime} W\left(\hat{G}_{q} x(t)+\hat{H}_{q} r\left(t_{0}\right)\right) \\
=x(t)^{\prime} \hat{G}_{q}^{\prime} W \hat{G}_{q} x(t)+2 r\left(t_{0}\right)^{\prime} \hat{H}_{q}^{\prime} W \hat{G}_{q} x(t) \\
\left.+r\left(t_{0}\right)^{\prime} \hat{H}_{q}^{\prime} W \hat{H}_{q} r\left(t_{0}\right)\right),
\end{gathered}
$$

the integral terms in (12) are given by

$$
\begin{aligned}
\int_{t_{0}}^{t_{1}} & \left(e_{T}(t)^{\prime} R e_{T}(t)+z(t)^{\prime} W z(t)+u(t)^{\prime} K u(t)\right) d t \\
& =\int_{t_{0}}^{t_{1}}\left(r ( t _ { 0 } ) ^ { \prime } \left(F^{\prime} C^{\prime} R C F+\hat{H}_{q}^{\prime} W \hat{H}_{q}\right.\right. \\
& \left.+\left(N^{\prime}-F^{\prime} C^{\prime} D_{q}^{\prime}\right) K\left(N-D_{q} C F\right)\right) r\left(t_{0}\right) \\
& \left.+x(t)^{\prime} P_{q} x(t)+c_{q}^{\prime} x(t)\right) d t,
\end{aligned}
$$


where $c_{q}^{\prime}$ is defined by

$$
c_{q}^{\prime}:=2 r\left(t_{0}\right)^{\prime}\left(-F^{\prime} C^{\prime} R \hat{C}_{q}+\hat{H}_{q}^{\prime} W \hat{G}_{q}+\left(N-C F D_{q}\right)^{\prime} K \tilde{C}_{q}\right)
$$

Those terms in (15) that depend on $x(t)$ are given by

$$
\begin{aligned}
& \int_{t_{0}}^{t_{1}}\left(x(t)^{\prime} P_{q} x(t)+c_{q}^{\prime} x(t)\right) d t= \\
& \quad \int_{t_{0}}^{t_{1}}\left(2 r\left(t_{0}\right)^{\prime} \hat{B}_{q}^{\prime} Q_{q} x(t)-\frac{d}{d t}\left(x^{\prime} Q_{q} x\right)+c_{q}^{\prime} x(t)\right) d t \\
& =\left(2 r\left(t_{0}\right)^{\prime} \hat{B}_{q}^{\prime} Q_{q}+c_{q}^{\prime}\right) \hat{A}_{q}^{-1}\left(e^{\hat{A}_{q} \Delta}-I\right) x\left(t_{0}\right) \\
& \quad+\left(2 r\left(t_{0}\right)^{\prime} \hat{B}_{q}^{\prime} Q_{q}+c_{q}^{\prime}\right) \hat{A}_{q}^{-1}\left(\hat{A}_{q}^{-1} e^{\hat{A}_{q} \Delta}-\right. \\
& \left.\quad \hat{A}_{q}^{-1}-\Delta I\right) \hat{B}_{q} r\left(t_{0}\right) \\
& -x\left(t_{0}\right)^{\prime} e^{\hat{A}_{q}^{\prime} \Delta} Q_{q} e^{\hat{A}_{q} \Delta} x\left(t_{0}\right) \\
& -2 r\left(t_{0}\right)^{\prime} \hat{B}_{q}^{\prime}\left(e^{\hat{A}_{q}^{\prime} \Delta}-I\right)\left(\hat{A}_{q}^{-1}\right)^{\prime} Q_{q} e^{\hat{A}_{q} \Delta} x\left(t_{0}\right) \\
& +x\left(t_{0}\right)^{\prime} Q_{q} x\left(t_{0}\right) \\
& +r\left(t_{0}\right)^{\prime} \hat{B}_{q}^{\prime}\left(e^{\hat{A}_{q}^{\prime} \Delta}-I\right)\left(\hat{A}_{q}^{-1}\right)^{\prime} Q_{q} . \\
& \hat{A}_{q}^{-1}\left(e^{\hat{A}_{q} \Delta}-I\right) \hat{B}_{q} r\left(t_{0}\right) .
\end{aligned}
$$

Combing the latest result and (14), we conclude that

$$
J=x\left(t_{0}\right)^{\prime} M_{q} x\left(t_{0}\right)-x\left(t_{0}\right)^{\prime} g_{q}+*
$$

where $*$ stands for additive terms that do not depend on the $x_{Q}\left(t_{0}\right)$. Such terms will thus not affect the optimal value for $x_{Q}\left(t_{0}\right)$. Since our optimization is only performed on the component $x_{Q}\left(t_{0}\right)$ of $x\left(t_{0}\right)$, we further re-write

$$
\begin{aligned}
J= & x_{Q}\left(t_{0}\right)^{\prime} M_{33}^{q} x_{Q}\left(t_{0}\right)+x_{Q}\left(t_{0}\right)^{\prime} \\
& \left(2\left(\left(M_{13}^{q}\right)^{\prime} x_{\mathrm{pl}}\left(t_{0}\right)+M_{32}^{q} \bar{x}\left(t_{0}\right)-g_{3}^{q}\right)+* .\right.
\end{aligned}
$$

Also $M_{33}^{q}$ is positive semi-definite, (16) is convex on $x_{Q}\left(t_{0}\right)$ and any vector $x_{Q}^{*}\left(t_{0}\right)$ satisfying the first order optimality condition

$$
M_{33}^{q} x_{Q}^{*}\left(t_{0}\right)=\frac{1}{2} g_{3}^{q}-\left(M_{13}^{q}\right)^{\prime} x_{\mathrm{pl}}\left(t_{0}\right)-M_{32}^{q} \bar{x}\left(t_{0}\right)
$$

provides a global minimum to $J$, see Boyd \& Vandenberghe (2004). In general, (17) may not be solvable, but in our specific problem it can be proved that a solution always exists Hespanha et al. (2007) and the minimum Euclidean norm solution to (17) is given by (13), see (Meyer, 2009, Chapter 5).

Remark 3.2: Theorem 3.1 provides an explicit formula for the optimal reset map. In practice, we cannot implement (13) since the left-hand side depends on the state of the process that may not be directly available. It turns out that the signal $\hat{x}:=\bar{x}+x_{e q}$ that can be directly obtained from the state of $\mathbf{C}(\sigma)$ converges exponentially to the process state $x_{\mathrm{pl}}(t)$ regardless of the control signal $u(t)$. To verify that this is so, we define the state estimation error $e=x_{\mathrm{pl}}-\hat{x}$ that, because of (3) and (1), with the matrices defined in (4)-(5), evolves according to $\dot{e}=\dot{x}_{\mathrm{pl}}-\dot{\hat{x}}=\dot{x}_{\mathrm{pl}}-\dot{\bar{x}}=(A-L C) e$ where $A-L C$ is a Hurwitz matrix. So, we can replace $x_{\mathrm{pl}}$ in (13) by $\hat{x}=\bar{x}+x_{e q}$ at the expense of introducing an error that vanishes to zero exponentially fast for every control input. This motivates the following reset map for (2):

$$
\begin{aligned}
& \psi_{Q}\left(x\left(t_{0}^{-}\right), p, q, r\left(t_{0}\right)\right):=V_{1}^{q} \Lambda_{q}^{-1}\left(U_{1}^{q}\right)^{\prime} . \\
&\left(\frac{1}{2} \hat{g}_{3}^{q}-\left(M_{32}^{q}+\left(M_{13}^{q}\right)^{\prime}\right) \bar{x}\left(t_{0}^{-}\right)\right)
\end{aligned}
$$

where $\hat{g}_{3}^{q}=g_{3}^{q}-2\left(M_{13}^{q}\right)^{\prime} F r\left(t_{0}\right)$. If one were to include exogenous disturbances and/or measurement noise in the process model (3), then the error $e(t)$ would not converge to zero and (18) would not be optimal. However, in this case one can select the matrix $L$ to minimize the steady-state mean-square error between $x_{\mathrm{pl}}$ and $\hat{x}$. This would correspond to a standard LQG estimation problem. As we shall see, our results do not depend on a specific choice for the matrix $L$, other that is must make $A-L C$ Hurwitz, so selecting $L$ based on a LQG optimization is a very reasonable option to minimize the loss of optimality due to discrepancies between $x_{\mathrm{pl}}$ and $\hat{x}$. Another option to minimize the influence of the state estimation error is to use a finite-time observer in parallel with the main observer used in the controller. In this case, one can use the result of finite-time observer in linear time-varying systems by Menold et al. (2003).

Remark 3.3 (robust optimization under parameter uncertainties): The optimal solution (13) involves $M_{33}^{q}$ matrices that are associated with the plant. In the presence of parameter uncertainty, one can use results from robust quadratic programming to compute the optimal solution, cf. Boyd \& Vandenberghe (2004): Let us consider a variation of (16) that includes uncertainty in the matrix $M_{33}^{q}$. We assume that this matrix belongs to a set $\mathcal{E}$ which is specified by a nominal value $M_{33}^{q^{0}}$ plus a bound on the eigenvalues of the deviation

$$
\mathcal{E}=\left\{M_{33}^{q} \mid-\gamma I \leq M_{33}^{q}-M_{33}^{q^{0}} \leq \gamma I\right\} .
$$

We define the robust quadratic program as

$$
\begin{aligned}
& \min \sup _{M_{33}^{q} \in \mathcal{E}} x_{Q}\left(t_{0}\right)^{\prime} M_{33}^{q} x_{Q}\left(t_{0}\right)+x_{Q}\left(t_{0}\right)^{\prime} \\
& \left(2\left(\left(M_{13}^{q}\right)^{\prime} x_{\mathrm{pl}}\left(t_{0}\right)+M_{32}^{q} \bar{x}\left(t_{0}\right)-g_{3}^{q}\right)+* .\right.
\end{aligned}
$$

For a given $x$, the supremum of $x^{\prime}\left(M_{33}^{q}-M_{33}^{q^{0}}\right) x$ over $\mathcal{E}$ is given by $\gamma x^{\prime} x$. Therefore, we can express the robust quadratic programming as

$$
\begin{aligned}
\min & x_{Q}\left(t_{0}\right)^{\prime}\left(M_{33}^{q^{0}}+\gamma I\right) x_{Q}\left(t_{0}\right)+x_{Q}\left(t_{0}\right)^{\prime} \\
& \left(2\left(\left(M_{13}^{q}\right)^{\prime} x_{\mathrm{pl}}\left(t_{0}\right)+M_{32}^{q} \bar{x}\left(t_{0}\right)-g_{3}^{q}\right)+*\right.
\end{aligned}
$$


leading to a reset map that minimizes (19).

\section{Input-to-State stability}

In section 3, we assumed that no further switching would occur in the interval $\left[t_{0}, t_{1}\right]$ but if there are further switchings, then the reset map (18) may destabilize the switched system even though all individual subsystems are asymptotically stable, e.g., Liberzon (2003). In this section, we show that we can achieve Input-to-StateStability (ISS) of the impulsive system (9)-(10) in the sense of Hespanha et al. (2008).

We first recall the following standard definitions: A continuous function $\alpha:[0, \infty) \rightarrow[0, \infty)$ is said to belong to class $\mathcal{K}$, when it is strictly increasing, and $\alpha(0)=0$. If $\alpha$ is also unbounded, then we say it is of class $\mathcal{K}_{\infty}$. A continuous function $\beta:[0, \infty) \times[0, \infty) \rightarrow[0, \infty)$ is of class $\mathcal{K} \mathcal{L}$ when $\beta(., t)$ is of class $\mathcal{K}$ for each fixed $t \geq 0$, and $\beta(r, t)$ decreases to 0 as $t \rightarrow \infty$ for each fixed $r \geq 0$.

It is often of interest to characterize input-to-state stability over classes of switching sequences. Suppose that $\mathcal{S}$ is the set of admissible switching signals. We say that the switching system (9)-(10) is uniformly input-to-state stable (ISS) over $\mathcal{S}$ if there exist functions $\beta \in \mathcal{K} \mathcal{L}$ and $\gamma \in \mathcal{K}_{\infty}$ such that for every initial condition, every reference signal $r(t)$, and every $\sigma \in \mathcal{S}$ the corresponding solution to (9)-(10) exists globally and satisfies

$$
|x(t)| \leq \beta\left(\left|x\left(t_{0}\right)\right|, t-t_{0}\right)+\gamma\left(\|r\|_{\left[t_{0}, t_{1}\right]}\right) \quad \forall t \geq t_{0}
$$

where $\|.\|_{I}$ denotes the supremum norm on an interval $I$. Moreover, let $\mathcal{S}_{\text {avg }}\left[\tau^{*}, N_{0}\right]$, with $\tau^{*}>0$ and $N_{0}>0$, denote the set of Average Dwell Time (ADT) switching sequences that satisfy

$$
N(t, s) \leq \frac{t-s}{\tau^{*}}+N_{0} \quad \forall t \geq s \geq t_{0}
$$

where $N(t, s)$ is the number of discontinuities of switching signal $\sigma$, in the open interval $(s, t)$. The constant $\tau^{*}$ is called average dwell time and $N_{0}$ the chatter bound, see Hespanha et al. (2008).

We further need the following concepts introduced in Hespanha et al. (2008). Consider the following system:

$$
\left\{\begin{array}{l}
\dot{x}(t)=f_{\sigma(t)}(x(t), r(t)), \quad t \neq t_{k} \\
x(t)=h_{\sigma(t)}\left(x\left(t^{-}\right), r\left(t^{-}\right)\right), t=t_{k}
\end{array}\right.
$$

We say that a function $V: \mathbb{R}^{n} \rightarrow \mathbb{R}$ is a candidate exponential ISS-Lyapunov function for (20) with rate coefficient $c, d \in \mathbb{R}$ if $V$ is locally Lipschitz, positive definite, radially unbounded, and satisfies

$$
\begin{gathered}
\nabla V(x) . f_{\sigma}(x, r) \leq-c V(x)+\mathcal{X}_{1}(|r|) \quad \forall x \text { a.e., } \forall r \\
V\left(h_{\sigma}\left(x^{-}, r^{-}\right)\right) \leq e^{-d} V(x)+\mathcal{X}_{2}(|r|) \quad \forall x, r
\end{gathered}
$$

for some functions $\mathcal{X}_{i} \in \mathcal{K}_{\infty}$ and all admissible switching signals $\sigma \in \mathcal{S}$. In $(21), " \forall x$ a.e." should be interpreted as "for every $x \in \mathbb{R}^{n}$ except, possibly, on a set of zero Lebesgue-measure in $\mathbb{R}^{n}$ ".

Theorem 4.1 Let $\bar{\tau}>0$ be given by

$$
\begin{aligned}
& \bar{\tau}=\inf _{c>0, Q_{1}, Q_{2}, Q_{3}>0} \tau \quad \text { subject to } \\
& {\left[\begin{array}{cc}
\left(e^{\tau c / 2}-1\right)\left(Q_{1}+Q_{2}\right) & \left(e^{\tau c / 2}-1\right)\left(-Q_{2}\right) \\
\left(e^{\tau c / 2}-1\right)\left(-Q_{2}\right) & \left(e^{\tau c / 2}-1\right) Q_{2}-\phi_{q}^{\prime} Q_{3} \phi_{q}
\end{array}\right] \geq 0} \\
& R_{q}\left(Q_{1}, Q_{2}, Q_{3}, c\right) \geq 0 \\
& \text { with } R_{q} \text { defined in }(24) \text { and } \phi_{q}:=-V_{1}^{q} \Lambda_{q}^{-1}\left(U_{1}^{q}\right)^{\prime}\left(\left(M_{13}^{q}\right)^{\prime}+\right. \\
& \left.M_{32}^{q}\right) . \text { For every average dwell-time switching sequence } \\
& \mathcal{S}_{\text {avg }}\left[\tau^{*}, N_{0}\right] \text { with } \tau^{*}>\bar{\tau}, \text { the switched system }(9)-(10) \\
& \text { with reset map (18) is ISS. }
\end{aligned}
$$

For fixed values of $\tau$ and $c,(23)$-(24) turn out to be linear matrix inequality conditions. One could solve the optimization problem of Theorem 4.1 by griding on $c, \tau$ (with an appropriate resolution) and checking the feasibility of (23)-(24). Thus, the solution would be the smallest $\tau$ for which (23)-(24) are feasible. One can also check if the closed-loop system remains ISS for a given $\tau$ by performing a line search on $c$ and checking the feasibility of (23)-(24) at each step.

Proof of Theorem 4.1 We define $V(x(t)):=x(t)^{\prime} Q x(t)$ as the Lyapunov function for the closed-loop system

$$
Q:=\Gamma^{\prime}\left[\begin{array}{ccc}
Q_{1} & 0 & 0 \\
0 & Q_{3} & 0 \\
0 & 0 & Q_{2}
\end{array}\right] \Gamma \quad \Gamma:=\left[\begin{array}{ccc}
I & 0 & 0 \\
0 & 0 & I \\
-I & I & 0
\end{array}\right]
$$

Straightforward algebra and (24) show that

$$
Q \hat{A}_{q}+\hat{A}_{q}^{\prime} Q \leq-c Q<0 .
$$

This implies that

$$
\nabla V(x) \cdot\left(\hat{A}_{\sigma(t)} x(t)+\hat{B}_{\sigma(t)} r(t)\right) \leq-c V(x)+b|x|\left|\hat{B}_{\sigma(t)} r(t)\right|
$$

from which (21) follows by appropriate choice of $\mathcal{X}_{1}(|r|)$. We will further need to show the inequality (22) is satisfied for any $\tau^{*}>\bar{\tau}$, from which $V(x)$ turns out to be a 
$R_{q}:=\left[\begin{array}{ccc}-(A-B K)^{\prime} Q_{1}-Q_{1}(A-B K)+c Q_{1} & -Q_{1} B C_{q} & Q_{1} B\left(K+D_{q} C\right) \\ -C_{q}^{\prime} B^{\prime} Q_{1} & -A_{q}^{\prime} Q_{3}-Q_{3} A_{q}+c Q_{3} & Q_{3} B_{q} C \\ \left(K+D_{q} C\right)^{\prime} B^{\prime} Q_{1} & C^{\prime} B_{q}^{\prime} Q_{3} & -(A-L C)^{\prime} Q_{2}-Q_{2}(A-L C)+c Q_{2}\end{array}\right] \geq 0$

candidate exponential Lyapunov function for the closedloop system. Let us consider the function

$$
K(s):=\min \left\{\frac{s}{2},|\bar{\tau} c| / 2 \inf _{|z| \geq s / 2, \nabla V(z) \neq 0} \frac{V(z)}{|\nabla V(z)|}\right\} .
$$

By construction $K$ is positive for $s \neq 0$, monotone nondecreasing, and radially unbounded. By a slight abuse of notation, we define $g(x, r):=\psi_{Q}(x, p, q, r)$ where the function $g$ depends on $p$ and $q$. For every $p, q \in \mathcal{P}$, pick arbitrary $(x, r)$ for which $v:=g(x, r)-g(x, 0)$ is small in the sense that

$$
|v|=|g(x, r)-g(x, 0)|<K(|g(x, 0)|) .
$$

Using the same procedure as in the proof of (Hespanha et al., 2008, Theorem 3), one can show that

$$
V(g(x, r)) \leq e^{\bar{\tau} c / 2} V(g(x, 0)) .
$$

Moreover, straightforward algebra shows that the constraint (23) of the minimization problem makes sure that

$$
V(g(x, 0)) \leq e^{\bar{\tau} c / 2} V(x)
$$

So one can conclude that (26) and (27) lead to

$$
V(g(x, r)) \leq e^{\bar{\tau} c} V(x)
$$

Suppose now that we pick $(x, r)$ for which $v$ is large in the sense that it satisfies

$$
|g(x, 0)| \leq K^{-1}(|v|)
$$

We now have

$$
\begin{gathered}
V(g(x, r))=V(v+g(x, 0)) \leq \alpha(|v|+|g(x, 0)|) \\
\leq \alpha \circ\left(i d+K^{-1}\right)(|v|) \leq \mathcal{X}_{2}(|r|)
\end{gathered}
$$

where $\alpha$ is a class $\mathcal{K}_{\infty}$ function with the property that $V(x) \leq \alpha(x)$. We also define $\mathcal{X}_{2}=\alpha \circ\left(i d+K^{-1}\right) \circ$ $\gamma$ with function $\gamma$ as the growth estimate of $g(x, r)=$ $g(x, 0)$. The existence of $\mathcal{X}_{2}$ is guaranteed in Hespanha et al. (2008). Combining (28) and (29), we conclude that (22) is satisfied for any $\tau^{*}>\bar{\tau}>0$. So $V(x)$ turns out to be a candidate exponential Lyapunov function for the closed-loop system, and according to (Hespanha et al., 2008, Theorem 1) the closed-loop system is ISS over $\mathcal{S}_{\text {avg }}\left[\tau^{*}, N_{0}\right]$.
Theorem 4.1 guarantees input-to-state stability of the closed-loop system when the average number of switches per unit of time is smaller than a specific value. If this is not the case then stability can still be achieved by adding a mild constraint to the optimization (16). This is the result of the next theorem.

Theorem 4.2 Let $c$ and positive definite matrices $Q_{1}, Q_{2}, Q_{3}$ satisfy the matrix inequality (24), and $\mathcal{S}_{\text {avg }}\left[\tau^{*}, N_{0}\right]$ denote average dwell time switching sequences with $\tau^{*}>0$ and $N_{0}>0$. The switched system (9)-(10) is ISS for a reset map (11) that is obtained by solving the following minimization problem

$$
\begin{aligned}
\min _{x_{Q}\left(t_{0}\right)} x_{Q}\left(t_{0}\right)^{\prime} M_{33}^{q} x_{Q}\left(t_{0}\right)+x_{Q}\left(t_{0}\right)^{\prime} . \\
\left(2\left(\left(M_{13}^{q}\right)^{\prime} x_{\mathrm{pl}}\left(t_{0}^{-}\right)+M_{32}^{q} \bar{x}\left(t_{0}^{-}\right)-g_{3}^{q}\right)\right)
\end{aligned}
$$

subject to

$$
\begin{aligned}
& x_{Q}\left(t_{0}\right)^{\prime} Q_{3} x_{Q}\left(t_{0}\right) \leq \\
& \left(e^{\tau^{*} c}-1\right)\left[x_{\mathrm{pl}}\left(t_{0}^{-}\right)^{\prime} \bar{x}\left(t_{0}^{-}\right)^{\prime}\right]\left[\begin{array}{cc}
Q_{1}+Q_{2} & -Q_{2} \\
-Q_{2} & Q_{2}
\end{array}\right]\left[\begin{array}{c}
x_{\mathrm{pl}}\left(t_{0}^{-}\right) \\
\bar{x}\left(t_{0}^{-}\right)
\end{array}\right] \\
& \quad+e^{\tau^{*} c} x_{Q}\left(t_{0}^{-}\right)^{\prime} Q_{3} x_{Q}\left(t_{0}^{-}\right)+\mathcal{X}_{2}\left(\left|r\left(t_{0}\right)\right|^{2}\right) .
\end{aligned}
$$

In the minimization problem of Theorem 4.2, the cost function (30) is the same as (16). The additional constraint (31) may lead to some increase in the value of the criterion (16), but it makes sure that the closed-loop system is ISS under the given ADT.

A natural choice for $c$ and $Q_{1}, Q_{2}, Q_{3}$ in Theorem 4.2 would be the constants that result from the optimization in the Theorem 4.1, since they minimize the allowable average dwell time.

Note that $M_{q}$ and consequently $M_{q}^{33}$ are positive semidefinite matrices, and the constraint is convex, so the minimization problem in Theorem 4.2 turns out to be convex. The convexity of the problem makes it computationally easy, however (30)-(31) should be solved at every switching time. Although this may require more online computational resources, the convex optimization problem can be solved very efficiently using the results of Mattingley \& Boyd (2010). 


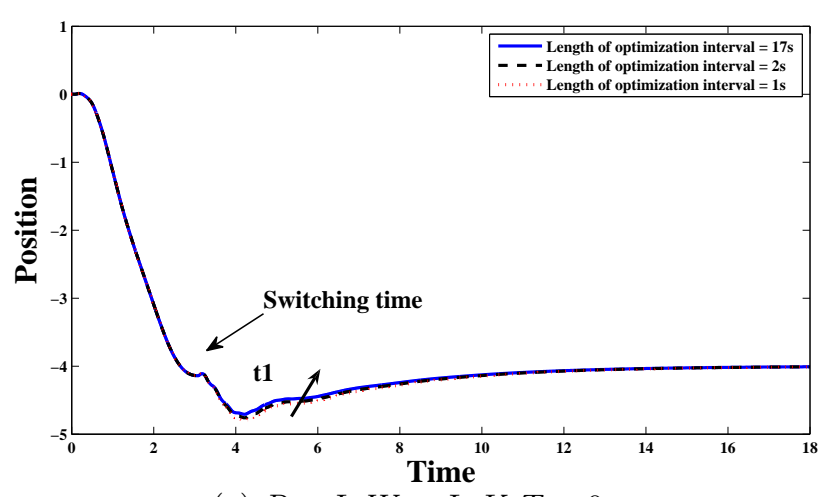

(a) $R=I, W=I, K, T=0$

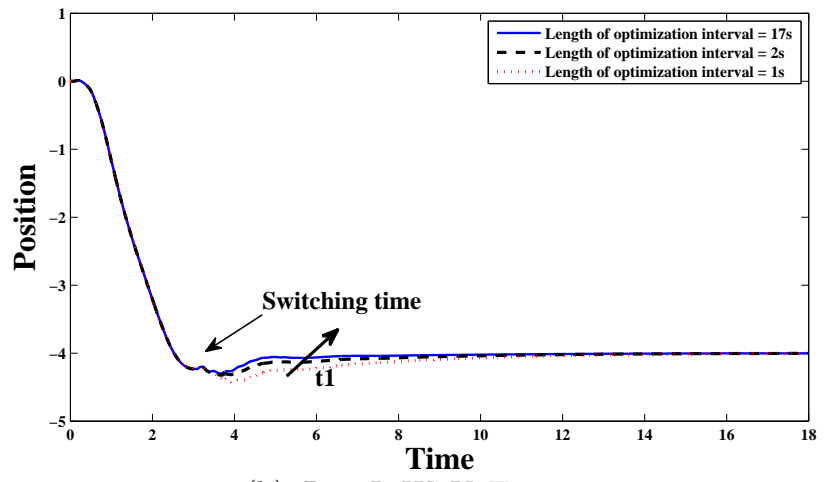

(b) $R=I, W, K, T=0$

Fig. 3. Transient responses for different weighting matrices and different lengths of the optimization interval $\left[t_{0}, t_{1}\right]$ $\left(t_{1}=4,5,20\right)$. In both plots there is a single controller switching at time $t_{0}=3 \mathrm{sec}$. By comparing the above plots, one can see how the penalty coefficient matrix $W$ for the output rate of change affects the transient responses. Details on the process and controllers being switched can be found in Section 5 .

Proof of Theorem 4.2 Using the same Lyapunov function $V(x)=x(t)^{\prime} Q x(t)$ as before, we have already shown that $(21)$ holds for $c>0$. Moreover, for any given Average Dwell Time switching sequences $\mathcal{S}_{\text {avg }}\left[\tau^{*}, N_{0}\right]$, we can always find $d<0$ such that $|d| \leq \tau^{*} c$; therefore the constraint (31) makes sure that for the given switching sequences we have

$$
V\left(x\left(t_{0}\right)\right) \leq e^{-d} V\left(x\left(t_{0}^{-}\right)\right)+\mathcal{X}_{2}\left(\left|r\left(t_{0}\right)\right|\right)
$$

at every switching time $t_{0}$. So $V(x)$ turns out to be a candidate exponential Lyapunov function for the closed-loop system, and according to (Hespanha et al., 2008, Corollary 1) the closed-loop system is ISS over $\mathcal{S}_{\text {avg }}\left[\tau^{*}, N_{0}\right]$.

Remark 4.3: It should be noted that choosing a large $\mathcal{X}_{2} \in \mathcal{K}_{\infty}$ in (31) leads to large ISS gain of the closedloop system. Since the ISS gain of the system is larger than $\max \left\{\mathcal{X}_{1}, \mathcal{X}_{2}\right\}$ in (21)-(22), one may choose $\mathcal{X}_{2}$ no larger than $\mathcal{X}_{1}$ in $(25)$.

Remark 4.4: When the process model (3) includes exogenous inputs such as measurement model and/or input disturbances, the results of Theorems 4.1 and 4.2 are still true for an input that includes the reference signal as well as these additional exogenous inputs. In this case, we would have to select $\mathcal{X}_{i}$ to make sure that (21)(22) hold for a more general input.

\section{Example}

In this section, we show simulation results for a closedloop system that switches between two controllers for a MIMO flexible beam. The matrices associated with the plant and controllers are given in Pour Safaei et al. (2010)
Figure 3 depicts the result of numerical simulations illustrating how varying the length of the optimization interval may influence the system's behavior. We can see that the transient response improves as we increase the length of optimization interval. Figure 4 (a) illustrates that we can achieve significantly better performance with the multicontroller proposed here than with the multicontroller proposed in Hespanha \& Morse (2002), which simply sets the left-hand side of (13) to zero. In Figure 4 (b), the results shown in Figure 4 (a) are compared to the case that there is no reset in the controller state.

Figure 5 shows the impact of using the reset map (18) instead of (13) in the presence of persistent noise and disturbance. As suggested in Remark 3.1, we selected the matrix $L$ to minimize the steady-state mean-square error between $x_{\mathrm{pl}}$ and $\hat{x}$.

Using the results of Theorem 4.1, we could show that the closed-loop system with reset map (18) remains ISS for average dwell time switching sequences $\mathcal{S}_{\text {avg }}\left[\tau, N_{0}\right]$ with $\tau$ as low as $10^{-16}$. Since matrices associated with (18) are computed offline, one can initialize the controllers by performing matrix multiplications at switching times.

\section{Conclusion and future directions}

In this paper, we showed that by finding optimal values for the initial controller state, one can achieve significantly better transients when switching between linear controllers for a not necessarily asymptotically stable MIMO linear process. The initialization was obtained by performing the minimization of a quadratic cost function of the tracking error, controlled output, and control signal. 


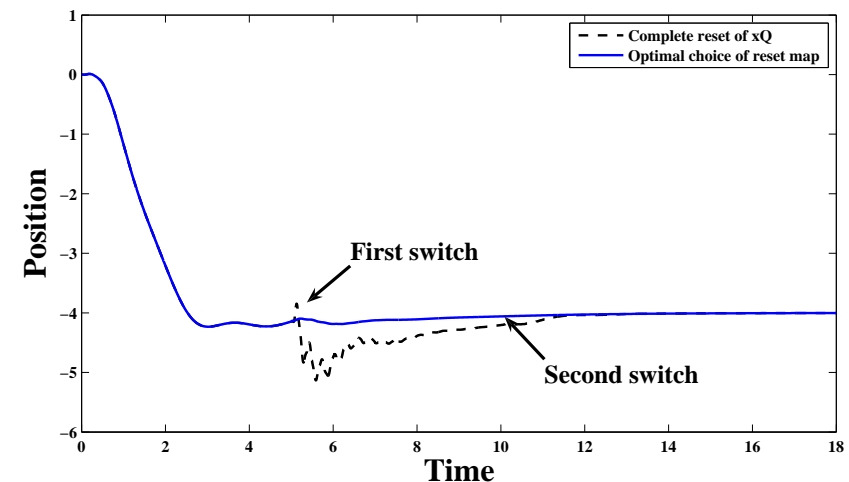

(a) Switching times: 5 and $10 \mathrm{sec}$

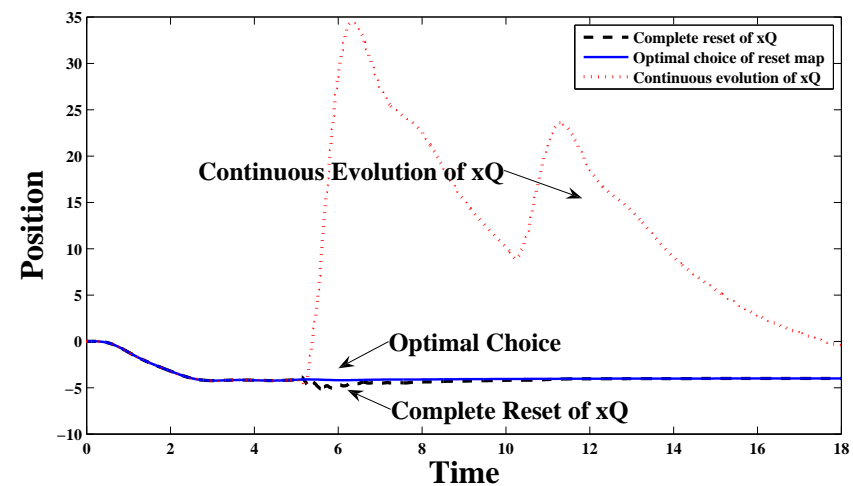

(b) Switching times: 5 and $10 \mathrm{sec}$

Fig. 4. Transient responses for the multicontroller proposed here and for the two alternative multicontrollers proposed in Hespanha \& Morse (2002) (for $R=I$ and $W, K, T=0$ ). The plots show the transients due to two control switchings at times 5 and 10 sec. The optimization intervals are $[5,10]$ and $[10,30]$. In Fig. (a) The "Optimal Choice" and "Complete Reset of $x_{Q}$ $\left(\psi_{Q}=0\right)$ " are shown, while in Fig. (b) the "Optimal Choice" and "Complete Reset of $x_{Q}$ " are compared to the "Continuous evolution of $x_{Q}\left(\psi_{Q}=I\right)$ ".

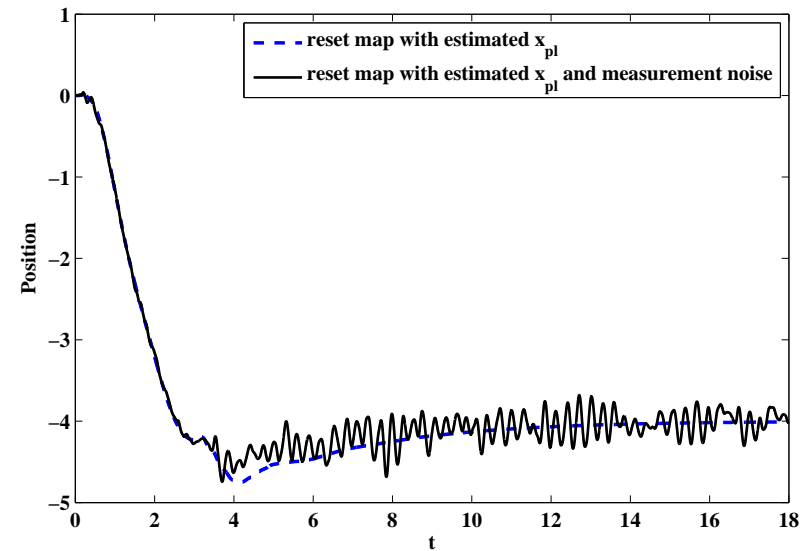

Fig. 5. This plot illustrates the impact of using the reset map 18), instead of the optimal map (13), in the presence of persistent measurement noise. The solid line shows the output trajectory when the output measurement is excited by a white noise with power 0.01 . There is a switching at $t_{0}=3$.

By suitable choice of realizations for the controllers, we guaranteed input-to-state stability of the closed-loop system when the average number of switches per unit of time is smaller than a specific value. If this is not the case, we showed that ISS property can be achieved under a mild constraint in optimization. A direction for future research is to consider the order of controller realization as a degree of freedom to guarantee closed-loop stability and to improve transient performance. Moreover, we assumed that the switching signal $\sigma(t)$ was provided externally however, in future, one may add a criterion for selecting appropriate switching times in the minimization of $J$ in (12). In many applications of switching control, destabilizing controllers are sometimes (temporarily) placed in the feedback loop. Such scenarios, which are not allowed in this paper, are also an important di- rection for future research.

\section{References}

Al-Shyoukh, I., \& Shamma, J. (2009). Switching supervisory control using calibrated forecasts. IEEE Transaction on Automatic Control, 54, 705-716.

Boyd, S., \& Barrat, C. (1991). Linear controller design: Limits of performance. New Jersey: Prentice Hall.

Boyd, S., \& Vandenberghe, L. (2004). Convex Optimization. New York, NY: Cambridge University Press.

Dullerud, G., \& Paganini, F. (1999). A course in robust control theory: a convex approach. New York: Springer.

Efimov, D., Cieslak, J., \& Henry, D. (2010a). Supervisory fault tolerant control via common lyapunov function approach. In Proc of Conference on Control and Fault Tolerance (pp. 582-587).

Efimov, D., Loria, A., \& Panteley, E. (2010b). Robust output stabilization: Improving performance via supervisory control. International Journal of Robust and Nonlinear Control, 21, 1219-1236.

Hespanha, J. P. (2009). Linear Systems Theory. Princeton, New Jersey: Princeton Press. ISBN13: 978-0-69114021-6.

Hespanha, J. P., Liberzon, D., \& Teel, A. R. (2008). Lyapunov conditions for input-to-state stability of impulsive systems. Automatica, 44, 2735-2744.

Hespanha, J. P., \& Morse, A. S. (2002). Switching between stabilizing controllers. Automatica, 38, 19051917.

Hespanha, J. P., Santesso, P., \& Stewart, G. E. (2007). Reset map design for switching between stabilizing controllers. In Proc. of the 46 th Conf. on Decision and Control (pp. $5634-5639$ ).

Liberzon, D. (2003). Switching in Systems and Control. Boston: Birkhäuser. 
Mattingley, J., \& Boyd, S. (2010). Real-time convex optimization in signal processing. Signal Processing Magazine, 27, 50-61.

Pour Safaei, F. R., Hespanha, J. P., \& Stewart, G. (2010). Quadratic optimization for controller inialization in multivariable switching systems. In Proc. of the 2010 American Control Conference (pp. 2511-2516).

Menold, P. H., Findeisen, R., \& Allgower, F. (2003). Finite time convergent observers for linear time-varying systems. In Proc. 11th Med. Conf. Control Autom. (MED03), Rhodos, Greece, (pp. 2365-2369).

Meyer, C. D. (2009). Matrix Analysis and Applied Linear Algebra. Philadelphia: Siam.

Stewart, G. E. (2012). A pragmatic approach to robust gain scheduling. To appear in 7th IFAC Symposium on Robust Control Design, .

Stewart, G. E., \& Dumont, G. A. (2006). Finite horizon based switching between stabilizing controllers. In Proc. of American Control Conference (pp. 15501556).

Turner, M., \& Walker, D. (2000). Linear quadratic bumpless transfer. Automatica, 36, 1089-1101.

$\mathrm{Xu}, \mathrm{X} .$, \& Antsaklis, P. J. (2004). Optimal control of switched systems based on parameterization of the switching instants. IEEE Transaction on Automatic Control, 49, 2-16.

Youla, D. C., Jabr, H. A., \& Bongiorno, J. J. (1976). Modern Wiener-Hopf design of optimal controllerspart II. the multivariable case. IEEE Transactions on Automatic Control, 21, 319-338.

Zheng, K., Basar, T., \& Benstman, J. (2007). $\mathrm{H}_{\infty}$ bumpless transfer under controller uncertainty. In Proc. of the 46th Conf. on Decision and Control (pp. 21292134).

Zheng, K., \& Benstman, J. (2009). Input/output structure of the infinite horizon LQ bumpless transfer and its implications for transfer operator synthesis. International Journal of Robust and Nonlinear Control, 20, 923-938. 